\title{
Long non-coding RNA MALAT1 is upregulated and involved in cell proliferation, migration and apoptosis in ovarian cancer
}

\author{
LIQIN WU $^{1}$, XIAOYU WANG ${ }^{2}$ and YUNA GUO ${ }^{3}$ \\ ${ }^{1}$ Department of Obstetrics and Gynecology, Daqing Oilfield General Hospital, Daqing, Heilongjiang 163001; \\ ${ }^{2}$ State Key Laboratory of Microbial Metabolism and School of Life Sciences and Biotechnology, Shanghai \\ Jiao Tong University; ${ }^{3}$ Department of Obstetrics, International Peace Maternity and Child Health \\ Hospital, Shanghai Jiao Tong University School of Medicine, Shanghai 200240, P.R. China
}

Received September 11, 2015; Accepted December 9, 2016

DOI: 10.3892/etm.2017.4304

\begin{abstract}
Ovarian cancer (OC) is the leading cause of mortality among gynecological malignancies. Although microRNAs are known to have a key regulatory role in $\mathrm{OC}$, the involvement of long non-coding RNAs in the disease is less established. Previous studies have demonstrated that metastasis-associated lung adenocarcinoma transcript 1 (MALAT1) is a tumor oncogene in many cancers, though its role in OC remains unclear. The present study reported that MALAT1 expression was markedly upregulated in OC, by knockdown of MALAT1 expression in vivo, using RNA interference (RNAi) with small-interfering RNA (siRNA). It was found that MALAT1 expression was positively correlated with the International Federation of Gynecology and Obstetrics stages of OC, tumor histological grade and lymph node metastasis. In addition, the differential MALAT1 levels between a human ovarian epithelial cell line (HOSE) and OC cell lines (ES-2, OVCAR3, SKOV3 and HO8910) were compared in vitro. Notably, MALAT1 was expressed to a high level in OC cells. Furthermore, exogenous knockdown of MALAT1 significantly repressed growth and migration of OC cells, and promoted their apoptosis. Collectively, the current findings suggest that upregulation of MALAT1 in OC may facilitate tumorigenesis and metastasis. Knockdown of MALAT1 expression has potential as a novel target for the diagnosis and therapy of OC.
\end{abstract}

Correspondence to: Mrs. Yuna Guo, Department of Obstetrics, International Peace Maternity and Child Health Hospital, Shanghai Jiao Tong University School of Medicine, 1961 Huashan Road Shanghai 200240, P.R. China

E-mail: guoyunaprofessor@sina.com

Key words: ovarian cancer, metastasis associated lung adenocarcinoma transcript 1 , metastasis, target

\section{Introduction}

Ovarian cancer $(\mathrm{OC})$ is the most prevalent cancer to occur in women worldwide, and is the leading cause of cancer-associated mortality in western countries (1). In the United States in 2012, there were 22,280 new cases of OC and 15,500 mortalities (2). There is a lack of definitive early symptoms and effective markers for OC diagnosis. Therefore, the prognosis for survival of $\mathrm{OC}$ patients is poor relative to other female malignancies, with a five-year survival rate for OC of $\sim 30 \%(3,4)$. The poor prognosis of patients with OC has been correlated with tumorigenesis, tumor progression and metastasis (5). Therefore, it is necessary to determine reliable biomarkers and the underlying molecular mechanisms of OC, as potential targets for therapy.

Long non-coding RNAs (lncRNAs) are macromolecules of $>200$ nucleotides in length which lack protein-coding capacity. LncRNAs have recently emerged as novel regulators of various cancers $(6,7)$. Studies have suggested that altered expression of lncRNAs may play key roles in the development of tumors, including those in breast cancer, lung cancer, bladder carcinoma and renal carcinoma. For example, in lung cancer cells, upregulation of the IncRNA HOX transcript antisense RNA (HOTAIR) enhances cell proliferation, migration and invasion (8). In gastric cancer, the lncRNA Linc00152 is involved in cell cycle arrest, migration, invasion, apoptosis and the epithelial to mesenchymal (ETM) transition of cancer cells (9). Similarly, the IncRNA Linc00617 has been found to have oncogenic activity in breast cancer (10). Furthermore, oncogenic lncRNA maternally expressed 3 regulates the tumor growth factor- $\beta$ signaling pathway via the formation of RNA-DNA triplex structures (11).

Metastasis-associated lung adenocarcinoma transcript 1 (MALAT1) was among the first cancer-associated lncRNAs to be reported (12), where it was found to be expressed at high levels in non-small cell lung cancer $(13,14)$. To date, evidence demonstrates that MALAT1 plays a key role in the progression of various cancers, including cervical cancer (15), osteosarcoma (16), breast cancer (17) and colorectal cancer (18). However, the expression and function of MALAT1 are currently unknown in OC. 
In the present study, the clinical significance and function of MALAT1 in OC tissues was investigated. OC cell proliferation, migration and apoptosis were determined by knockdown of MALAT1 expression in vivo, using RNA interference (RNAi) with small-interfering RNA (siRNA). The data obtained highlight the significance of MALAT1 in OC progression and metastasis.

\section{Materials and methods}

Tissue samples and patient information. A total of 42 surgical tissue samples of $\mathrm{OC}$, along with matched normal ovarian tissues, from the same patient sample and located $2 \mathrm{~cm}$ away from visible OC lesions, were collected from the Department of Obstetrics and Gynecology at the Daqing Oilfield General Hospital (Daqing, China) between January 2010 and December 2014. Tissue samples were immediately immersed in RNAlater (Qiagen GmbH, Hilden, Germany) for $30 \mathrm{~min}$ and subsequently transferred into liquid nitrogen for cryopreservation until needed. No patients had received radiotherapy or chemotherapy therapy prior to surgery. The section tissues were stained with hematoxylin and eosin stain in order to determine tumor grade and stage, according to the criteria of the International Federation of Gynecologists and Obstetricians (FIGO) (19). The present study was permitted by the ethics committee of the Daqing Oilfield General Hospital and informed consent was obtained from all patients.

Cell lines and cell culture. The human ovarian epithelial cell line (HOSE), used as a control, and OC cells (ES-2, OVCAR3, SKOV3 and HO8910) were bought from American Type Culture Collection (Manassas, VA, USA). Cells were cultured in RPMI-1640 medium supplemented with $10 \%$ (v/v) fetal calf serum (Invitrogen; Thermo Fisher Scientific, Inc., Waltham, $\mathrm{MA}, \mathrm{USA}$ ) at $37^{\circ} \mathrm{C}$ in a humidified chamber with $5 \% \mathrm{CO}_{2}$ for $24 \mathrm{~h}$.

Reverse transcription-quantitative polymerase chain reaction (RT-qPCR). Total RNA was extracted from ovarian cell lines using TRIzol reagent (Invitrogen; Thermo Fisher Scientific) and purified with an RNeasy Maxi kit (Qiagen $\mathrm{GmbH}$ ) according to the manufacturers' protocols. The cDNAs were synthesized using $1 \mu \mathrm{g}$ total RNA according to Prime-Script RT-PCR kit (Takara Biotechnology Co., Ltd., Dalian, China) according to the manufacturer's protocol. The RT-qPCR experiments were conducted on an ABI PRISM 7700 Sequence Detection System (Applied Biosystems; Thermo Fisher Scientific, Inc.) using a SYBR Premix EX Taq II PCR kit (Takara Biotechnology Co., Ltd.) according to the manufacturer's protocol. The sequences for the MALAT1 primers were as follows: Forward, 5'-AAAGCAAGGTCT CCCCACAA-3', and reverse, 5'-GGTCTGTGCTAGATC AAAAGGCA-3'. GAPDH was used as an endogenous loading control. The GAPDH primers were as follows: Forward, 5'-GAAGGTGAAGGTCGGAGTC-3' and reverse, 5'-GAA GATGGTGATGGGATTTC-3'. All experiments were performed in triplicate. Relative expression level of MALAT1 was normalized with GAPDH and quantified using the $\Delta \Delta \mathrm{C}_{\mathrm{q}}$ method (20).
Cell transfection and cell proliferation assay. The following sequence of siRNA oligonucleotides (siMALAT1) was used to knockdown MALAT1 expression: 5'-CACAGGGAAAGCGAGUGGUUGGUA-3'. The sequence of the non-coding (NC) control siRNA (siNC) was 5'-UUCUCCGAACGUGUCACGU-3'. For the in vitro study, Lipofectamine ${ }^{\circledR} 2000$ transfection reagent (Invitrogen; Thermo Fischer Scientific, Inc.) was used to transfect either siMALAT1 or siNC into OVCAR3 and HO8910 cells, according to the manufacturer's protocol. After $48 \mathrm{~h}$ of transfection, the efficiency of MALAT1 knockdown was evaluated by RT-qPCR analysis, as described above, prior to in vitro cell function experiments.

Cell proliferation assay. Cell proliferation was detected using an MTT assay in vitro. OC cell lines were cultured as described above and were seeded with RPMI-1640 at a density of $\sim 8,000$ cells per well in a 96-well plate and transfected with $10 \mathrm{nM}$ siMALAT1 or siNC for $12,24,48$ and $72 \mathrm{~h}$ at $37^{\circ} \mathrm{C}$ in a humidified chamber with $5 \% \mathrm{CO}_{2}$, using Lipofectamine 2000 reagent (Invitrogen; Thermo Fisher Scientific, Inc.) according to the manufacturer's protocol. Following transfection, cells were treated with $30 \mu \mathrm{l}$ MTT $(5 \mathrm{mg} / \mathrm{ml})$ per well and incubated for $4 \mathrm{~h}$ at $37^{\circ} \mathrm{C}$ in a humidified chamber at $5 \% \mathrm{CO}_{2}$. The crystals generated were dissolved in $150 \mu 1$ dimethyl sulfoxide solvent. Absorbance was measured at $490 \mathrm{~nm}$ with an enzyme immunoassay analyzer (Bio-Rad Laboratories, Inc., Hercules, CA, USA) immediately following solvent addition.

Cell migration assay. A wound scratch assay was used to evaluate the migration abilities of OC cells. OC cell lines were cultured as described above. OVCAR3 and HO8910 cells were seeded with RPMI-1640 at a density of approximately $5 \times 10^{6}$ cells per well in a 6 -well plate and transfected with $10 \mathrm{nM}$ siMALAT1 or siNC as above. Wounds were made with 100- $\mu 1$ pipette tips when cell confluence reached 50-75\%. Cells were then washed with phosphate-buffered saline (PBS) to remove residual free-floating cells and debris. Following this, incubation of the cells was continued in a humidified chamber at $37^{\circ} \mathrm{C}$ with $5 \% \mathrm{CO}_{2}$ for $6 \mathrm{~h}$ and a $100-\mu \mathrm{l}$ pipette tip was used to make a wound in the cell culture. Different stages of wound healing were observed along the wound line and cell migration was measured using a standard caliper. Representative images were captured using a Leica DM LB2 light microscope digital camera system (Leica Microsystems GmbH, Wetzlar, Germany).

Flow cytometry. OC cell lines were cultured as described above, and $\sim 5 \times 10^{6}$ OVCAR3 and HO8910 cells per well were cultured in 6 -well plates at $37^{\circ} \mathrm{C}$ and transfected with $100 \mathrm{nM}$ siMALAT1 or siNC within 24 h using Lipofectamine ${ }^{\circledR} 2000$ reagent (Invitrogen; Thermo Fisher Scientific, Inc.) according to the manufacturer's protocol. Flow cytometry was performed as previously described (21). At $48 \mathrm{~h}$ post-transfection, cells were collected using trypsin reagent (Sigma-Aldrich, Merck KGaA, Darmstadt, Germany) and washed three times with cold PBS, then re-suspended in $100 \mu \mathrm{l}$ of $1 \mathrm{X}$ binding buffer (Invitrogen; Thermo Fisher Scientific, Inc.). Following resuspension, $5 \mu \mathrm{l}$ propidium iodide and $5 \mu \mathrm{l}$ Annexin V-fluorescein isothiocyanate stain (Invitrogen; Thermo Fisher Scientific,Inc.) 
was added to each well suspension. The apoptotic rate of the stained cells was analyzed using flow cytometry (Beckman Coulter, Inc., Brea, CA, USA) within 30 min of staining.

Statistical analysis. The results are expressed as the mean \pm standard error of the mean. The differences between cell lines were analyzed using the two-sided Student's t-test or the analysis of variance test using SPSS 16.0 statistical software (SPSS, Inc., Chicago, IL, USA). P<0.05 was considered to indicate a statistically significant difference.

\section{Results}

MALATl was significantly increased in OC tissues and correlated with poor prognosis. The expression level of MALAT1 in OC tissues obtained from 42 patients and in matched non-cancerous tissues was analyzed using RT-qPCR. Relative expression of MALAT1 is presented in Fig. 1A. The results demonstrated that MALAT1 expression in OC tissues was higher compared with normal ovary tissues. MALAT1 expression in OC was significantly upregulated relative to its expression level in normal ovary tissues $(\mathrm{P}=0.001$; Fig. 1B). These data were consistent with the level of MALAT1 in other cancers such as breast cancer, non-small cell lung cancer and esophageal squamous cell carcinoma, suggesting MALAT1 may also function as an oncogene in OC.

For the clinicopathological correlation analysis, the 42 OC patients were classified into two groups with the mean expression of MALAT1 as the threshold (3.27 relative to GAPDH): A high MALAT1 expression group $(n=36)$; and a low MALAT1 expression group $(n=6)$. As depicted in Table I, higher relative expression was positively correlated with advanced histological grade (G3; P<0.001), higher FIGO stages (III-IV; $\mathrm{P}=0.001$ ) and lymph node metastasis $(\mathrm{P}<0.001)$. Expression was not correlated with age and tumor size.

MALAT1 was upregulated in OC cells compared with normal ovarian epithelial cells, and RNAi downregulated MALATI expression. The MALAT1 expression level in OC cell lines was also detected. The expression of MALAT1 was found to be notably increased in the four OC cell lines examined (ES-2, OVCAR3, SKOV3 and HO8910), as compared with the normal ovarian cell line HOSE (all $\mathrm{P}<0.05$; Fig. $2 \mathrm{~A}$ ).

Furthermore, to illustrate the function of MALAT1 in OC, siMALAT1 or control siNC were transfected into OVCAR3 and HO8910 cells. RT-qPCR was conducted to detect the transfection efficiency. The expression levels of MALAT1 in siMALAT1 samples were reduced by 0.35 - and 0.28 -fold in OVCAR3 and HO8910 cells, respectively, relative to the siNC samples $(\mathrm{P}<0.05$; Fig. 2B).

Exogenous knockdown of MALAT1 suppressed OC cell proliferation in vitro. To identify the potential role of MALAT1 in $\mathrm{OC}$, the inhibitory effect of MALAT1 knockdown on OC cell proliferation was measured. Cell proliferation rates were detected by the MTT assay after transfection of siMALAT1 or siNC into HO8910 and OVCAR3 cells. As shown in Fig. 3, knockdown of MALAT1 significantly repressed OC cell proliferation relative to siNC transfected cells $(\mathrm{P}<0.05)$. These
Table I. Association of MALAT1 expression with clinicopathological characteristics in ovarian cancer patients.

\begin{tabular}{|c|c|c|c|c|}
\hline \multirow[b]{2}{*}{ Variables } & \multirow[b]{2}{*}{ Cases } & \multicolumn{2}{|c|}{$\begin{array}{l}\text { MALAT1 } \\
\text { expression }\end{array}$} & \multirow[b]{2}{*}{ P value } \\
\hline & & Low & High & \\
\hline Age (years) & & & & 1.000 \\
\hline$<50$ & 19 & 3 & 16 & \\
\hline$\geq 50$ & 23 & 3 & 20 & \\
\hline Tumor size & & & & 0.663 \\
\hline$<1 \mathrm{~cm}$ & 21 & 2 & 19 & \\
\hline$\geq 1 \mathrm{~cm}$ & 21 & 4 & 17 & \\
\hline Histological grade & & & & $<0.001$ \\
\hline $\mathrm{G} 1-\mathrm{G} 2$ & 5 & 5 & 0 & \\
\hline G3 & 37 & 1 & 36 & \\
\hline FIGO stage & & & & 0.001 \\
\hline I-II & 5 & 4 & 1 & \\
\hline III-IV & 37 & 2 & 35 & \\
\hline Lymph node metastasis & & & & $<0.001$ \\
\hline Absent & 11 & 6 & 5 & \\
\hline Present & 31 & 0 & 31 & \\
\hline
\end{tabular}

MALAT1, metastasis associated lung adenocarcinoma transcript 1; FIGO, International Federation of Gynecologists and Obstetricians.
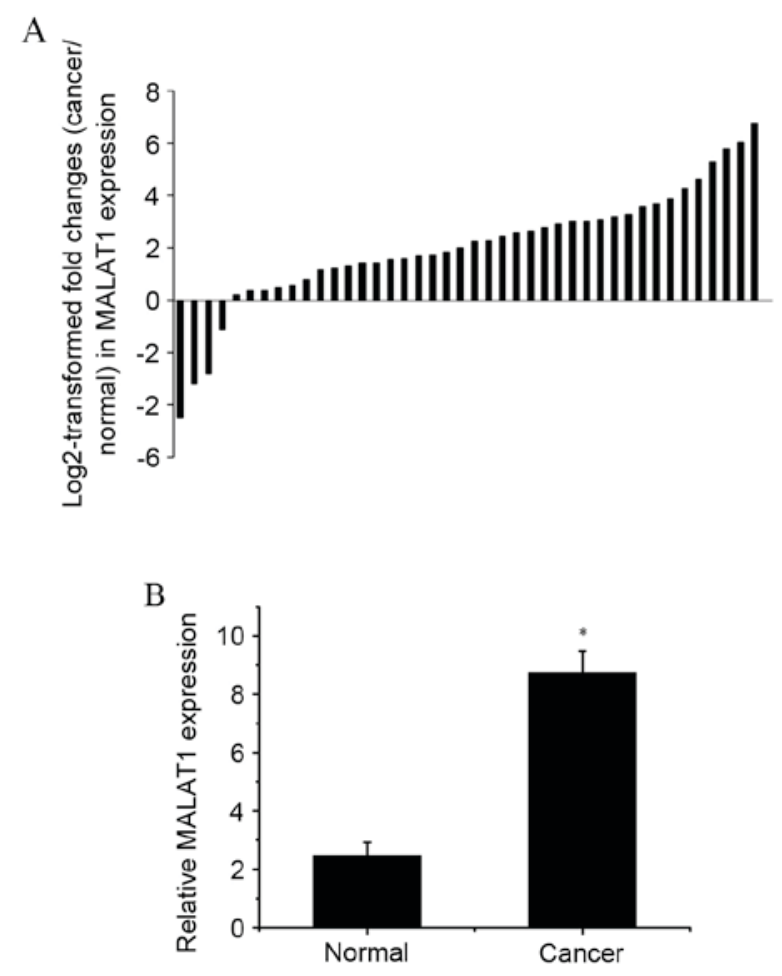

Figure 1. MALAT1 expression was significantly increased in OC tissues. (A) MALAT1 expression level in OC tissues obtained from 42 patients relative to matched normal ovary tissues. The data were analyzed using the $\Delta \Delta \mathrm{C}_{\mathrm{q}}$ method and are displayed in $\log _{2}$ (cancer/normal) format. (B) Reverse transcription-quantitative polymerase chain reaction analysis of MALAT1 level in OC tissues and matched normal ovary tissues. Results are expressed as the mean \pm standard error of the mean $(n=3)$. $P=0.001$. MALAT1, metastasis associated lung adenocarcinoma transcript 1; OC, ovarian cancer. 
A

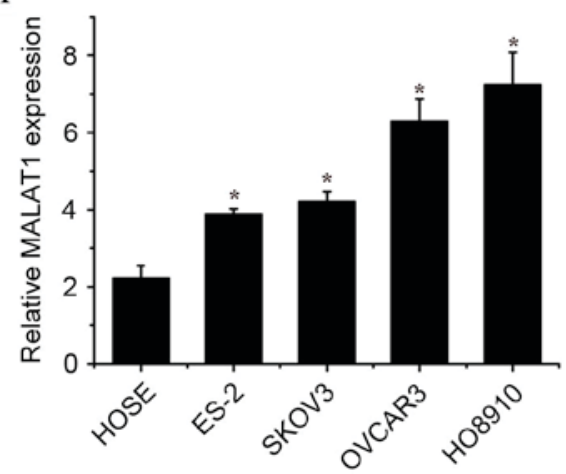

B

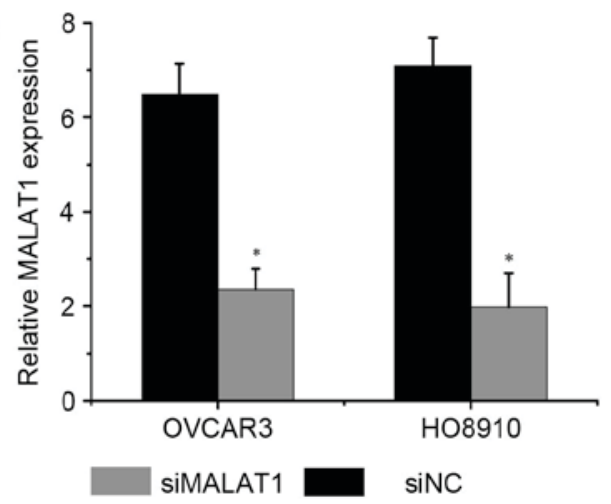

Figure 2. MALAT1 was upregulated in ovarian cancer cells relative to normal ovarian epithelial cells. (A) The relative expression level of MALAT1 was detected by reverse transcription-quantitative polymerase chain reaction analysis in four ovarian cancer cell lines (ES-2, OVCAR3, SKOV3 and HO8910) and a normal ovarian epithelial cell line (HOSE). * $\mathrm{P}<0.05$ vs. HOSE. (B) The fold changes of MALAT1 expression in HO8910 and OVCAR3 cells treated with siMALAT1 or siNC were analyzed $48 \mathrm{~h}$ post-transfection. Data are presented as the mean \pm standard error of the mean. Experiments were performed in triplicate. "P<0.05 vs. siNC transfectants. MALAT1, metastasis associated lung adenocarcinoma transcript 1; siMALAT1, MALAT1 small interfering RNA; siNC, non-coding small interfering RNA.

A
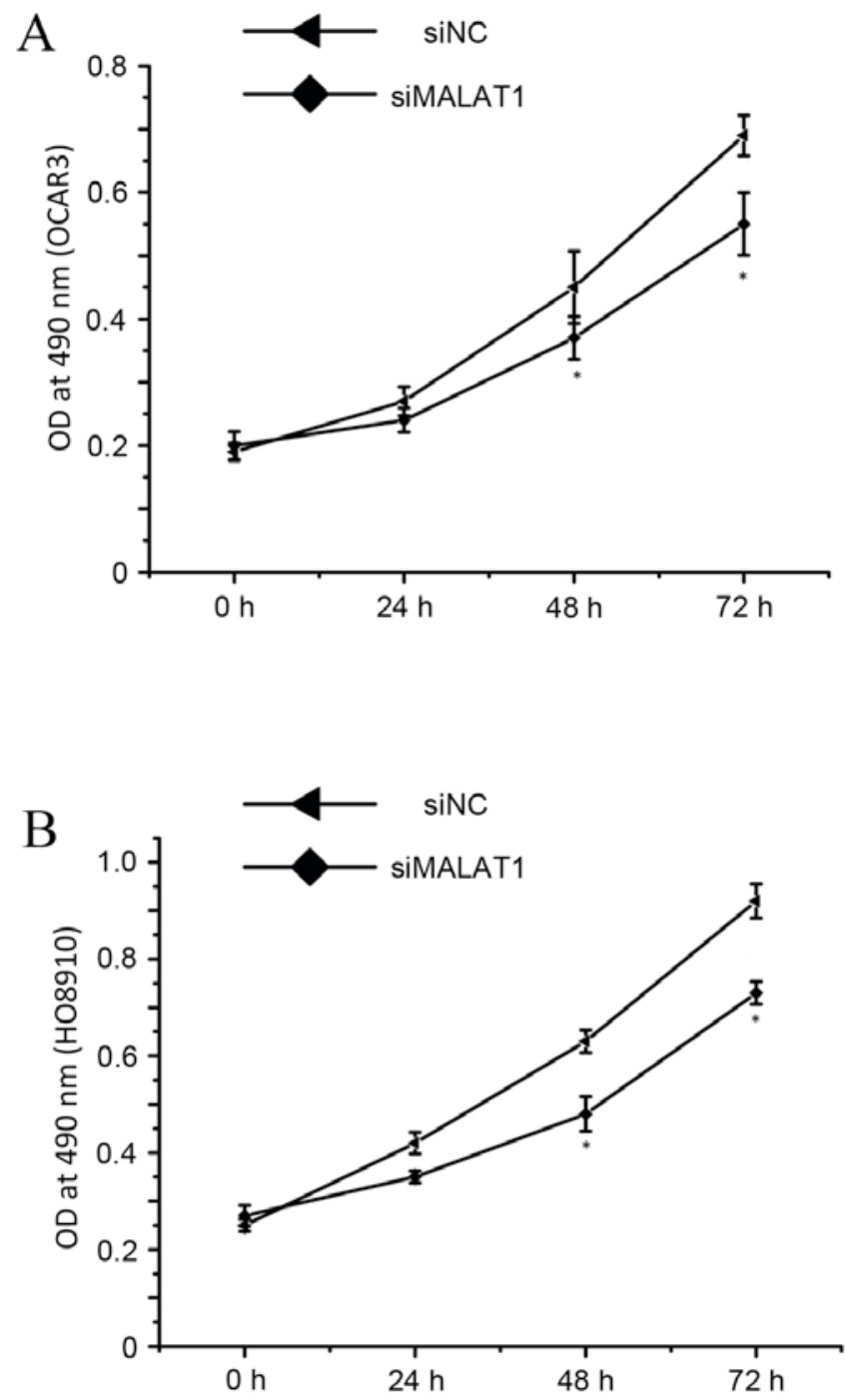

Figure 3. Exogenous knockdown of MALAT1 suppressed ovarian cancer cell proliferation in vitro. The MTT assay revealed significant inhibition of cell proliferation in siMALAT1 transfectants relative to siNC transfectants of the (A) OVCAR3 and (B) HO8910 cell lines. "P<0.05. MALAT1, metastasis associated lung adenocarcinoma transcript 1; siMALAT1, MALAT1 small interfering RNA; siNC, non-coding small interfering RNA. results indicate that knockdown of MALAT1 inhibits the proliferation of OC cells in vitro.

Exogenous knockdown of MALAT1 repressed OC cell migration in vitro. To test the role of MALAT1 in OC cell metastasis, the migratory ability of OC cells was examined by a wound scratch assay. This involved siRNA knockdown of MALAT1 in HO8910 and OVCAR3 cells. The results demonstrated that following incubation for $24 \mathrm{~h}$, wound widths in HO8910 and OVCAR3 cells transfected with siMALAT1 were notable larger than in those transfected with siNC (Fig. 4A). Knockdown of MALAT1 caused significant inhibition of cell migration in both HO8910 $(\mathrm{P}=0.01)$ and OVCAR3 $(\mathrm{P}=0.04)$ cells (Fig. 4B). Therefore, it was concluded that knockdown of MALAT1 may suppress OC cell migration in vitro.

Exogenous knockdown of MALAT1 induces OC cell apoptosis in vitro. To determine the effect of MALAT1 on OC cell apoptosis, a flow cytometry assay was performed to detect the apoptotic rates of HO8910 and OVCAR3 cells at $48 \mathrm{~h}$ post-transfection (Fig. 5A). It was found that the apoptotic rates of HO8910 cells treated with siMALAT1 and siNC were 10.14 and $4.27 \%$, respectively $(\mathrm{P}=0.02)$, while those for OVCAR3 cells were 9.51 and $3.32 \%$, respectively ( $\mathrm{P}=0.001$; Fig. $5 \mathrm{~B}$ ). Collectively, the results suggest that MALAT1 functions as an oncongene in $\mathrm{OC}$ by regulating cell proliferation, migration and apoptosis.

\section{Discussion}

Increasing evidence indicates that eukaryotic genomes and transcriptomes are the precursors for a number of non-coding RNAs (22), in addition to their established roles as protein-coding genes. Studies have highlighted key regulatory roles of lncRNAs in carcinogenesis and have suggested that some lncRNAs may be potential diagnostic and therapeutic targets in the treatment of OC $(23,24)$. For example, evidence shows that MALAT1 is downregulated in breast cancer, and that knockdown of MALAT1 induces the EMT program, via pathways involving phosphatidylinositide-3 kinase (17). It has 
A OVCAR3
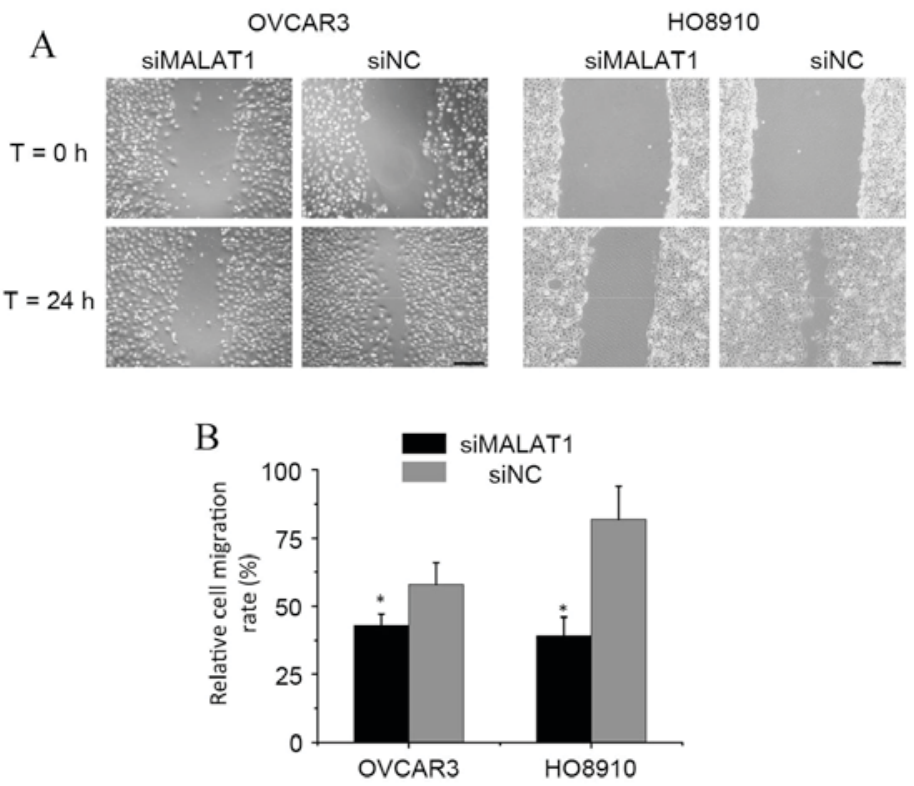

Figure 4. Exogenous knockdown of MALAT1 repressed ovarian cancer cell migration in vitro. (A) Microscopic analysis of the wound assay enabled visualization of the inhibited cell migration in siMALAT1-transfectants relative to the siNC control. Scale bar, $5 \mu \mathrm{m}$. (B) The wound scratch assay found significant inhibition of cell migration in siMALAT1-treated cells relative to siNC-treated cells of the OVCAR3 and HO8910 cell lines. * P<0.05 vs. siNC. MALAT1, metastasis associated lung adenocarcinoma transcript 1; siMALAT1, MALAT1 small interfering RNA; siNC, non-coding small interfering RNA.

A

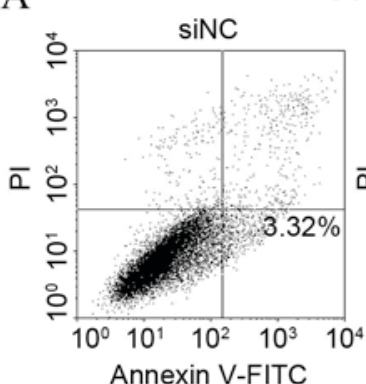

Annexin V-FITC
OVCAR3

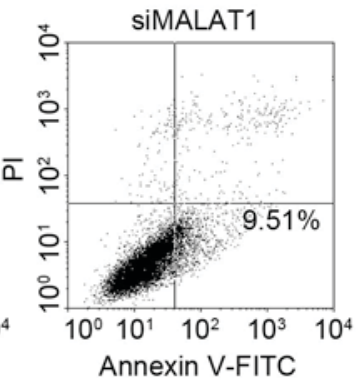

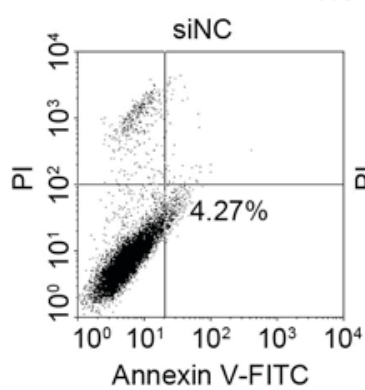

Annexin V-FITC
HO8910

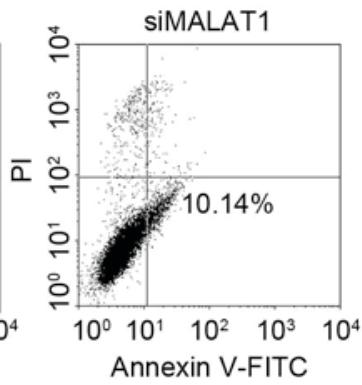

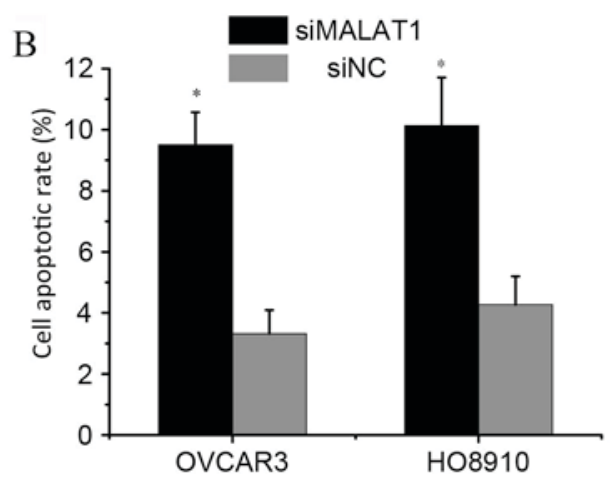

Figure 5. Exogenous knockdown of MALAT1 induces ovarian cancer cell apoptosis in vitro. (A) Apoptosis of OVCAR3 and HO8910 cells transfected with siMALAT1 or siNC was analysed by flow cytometry. The percentage displayed on the histograms indicates apoptotic rate. (B) Comparison of the apoptotic rates of cells transfected with siMALAT1 or siNC. Data are presented as the mean \pm standard error of the mean ( $\mathrm{n}=3$ ). ${ }^{*} \mathrm{P}<0.05 \mathrm{vs}$. siNC. MALAT1, metastasis associated lung adenocarcinoma transcript 1; siMALAT1, MALAT1 small interfering RNA; siNC, non-coding small interfering RNA.

also been found that the intronic lncRNA prostate cancer-associated 3 regulates protein prune homolog 2, a human prostate cancer suppressor (25). Furthermore, the IncRNA HOTAIR promotes cell migration and invasion through downregulation of RNA-binding motif protein 38 in hepatocellular carcinoma cells, with increased HOTAIR expression showing promise as a novel biomarker for the diagnosis and/or prognosis of hepatocellular carcinoma (26). These data highlight the roles and clinical significance of lncRNAs in carcinogenesis.

MALAT1 is an evolutionarily conserved lncRNA that does not undergo translation into protein (16). Nevertheless, previous findings suggest that MALAT1 regulates the 
alternative splicing of pre-mRNAs by controlling the expression of serine/arginine splicing factors, while also having a key role in tumorigenesis (27). Furthermore, research has indicated that knockdown of MALAT1 alters the process of metastasis, as a critical event in cancer biology (28). In addition, MALAT1 has been found to promote aggressive renal cell carcinoma (RCC) through the chromatin methyltransferse enhancer of zeste homolog 2, and interaction with microRNA-205, resulting in oncogenic activity (29).

Consistent with previous data $(28,29)$, the results of the present study found that MALAT1 was significantly expressed in OC tissue samples and cell lines. Furthermore, high MALAT1 expression was more strongly correlated with advanced histological grade, high FIGO stage and lymph node metastasis. These findings suggest that MALAT1 may act as an oncogene in OC.

To investigate the biological function of MALAT1 in OC cell lines, siRNA knockdown of MALAT1 in HO8910 and OVCAR3 cells was performed. Using the MTT assay, it was found that depletion of MALAT1 significantly inhibited cell proliferation in vitro. In addition, cell migration and apoptosis were also suppressed by exogenous MALAT1 knockdown in vitro. These results suggest that MALAT1 functions as a novel oncogene that regulates OC cell proliferation, migration and apoptosis.

In summary, the present study indicates a key role for MALAT1 in OC development, including tumor growth, migration and apoptosis, suggesting that MALAT1 may be a novel biomarker for OC prognosis and treatment. However in the present study, the number of samples of $\mathrm{OC}$ was limited to 42 , whereas more samples may allowed for the verification of its potential oncogenic activities in future studies.

\section{References}

1. Siegel R, Naishadham D and Jemal A: Cancer statistics, 2012. CA Cancer J Clin 62: 10-29, 2012.

2. Suh DH, Kim JW, Kim K, Kim HJ and Lee KH: Major clinical research advances in gynecologic cancer in 2012. J Gynecol Oncol 24: 66-82, 2013.

3. Conover CA, Hartmann LC, Bradley S, Stalboerger P, Klee GG, Kalli KR and Jenkins RB: Biological characterization of human epithelial ovarian carcinoma cells in primary culture: The insulin-like growth factor system. Exp Cell Res 238: 439-449, 1998

4. Jelovac D and Armstrong DK: Recent progress in the diagnosis and treatment of ovarian cancer. CA Cancer J Clin 61: 183-203, 2011.

5. Lengyel E: Ovarian cancer development and metastasis. Am J Pathol 177: 1053-1064, 2010.

6. Nagano $T$ and Fraser P: No-nonsense functions for long noncoding RNAs. Cell 145: 178-181, 2011.

7. Wilusz JE, Sunwoo H and Spector DL: Long noncoding RNAs: Functional surprises from the RNA world. Genes Dev 23: 1494-1504, 2009.

8. Zhou C, Ye L, Jiang C, Bai J, Chi Y and Zhang H: Long noncoding RNA HOTAIR, a hypoxia-inducible factor-1a activated driver of malignancy, enhances hypoxic cancer cell proliferation, migration, and invasion in non-small cell lung cancer. Tumour Biol 36: 9179-9188, 2015.

9. Zhao J, Liu Y, Zhang W, Zhou Z, Wu J, Cui P, Zhang Y and Huang G: Long non-coding RNA Linc00152 is involved in cell cycle arrest, apoptosis, epithelial to mesenchymal transition, cell migration and invasion in gastric cancer. Cell Cycle 14: 3112-3123, 2015

10. Li H, Zhu L, Xu L, Qin K, Liu C, Yu Y, Su D, Wu K and Sheng Y: Long noncoding RNA linc00617 exhibits oncogenic activity in breast cancer. Mol Carcinog 56: 3-17, 2017.
11. Mondal T, Subhash S, Vaid R,Enroth S, Uday S, Reinius B, Mitra S, Mohammed A, James AR, Hoberg E, et al: MEG3 long noncoding RNA regulates the TGF- $\beta$ pathway genes through formation of RNA-DNA triplex structures. Nat Commun 6: 7743, 2015.

12. Gutschner T, Hämmerle M and Diederichs S: MALAT1-a paradigm for long noncoding RNA function in cancer. J Mol Med (Berl) 91: 791-801, 2013.

13. Ji P, Diederichs S, Wang W, Böing S, Metzger R, Schneider PM, Tidow N, Brandt B, Buerger H, Bulk E, et al: MALAT-1, a novel noncoding RNA, and thymosin beta4 predict metastasis and survival in early-stage non-small cell lung cancer. Oncogene 22: 8031-8041, 2003

14. Gutschner T, Hämmerle M, Eissmann M, Hsu J, Kim Y, Hung G, Revenko A, Arun G, Stentrup M, Gross M, et al: The noncoding RNA MALAT1 is a critical regulator of the metastasis phenotype of lung cancer cells. Cancer Res 73: 1180-1189, 2013.

15. Guo F, Li Y, Liu Y, Wang J and Li G: Inhibition of metastasis-associated lung adenocarcinoma transcript 1 in CaSki human cervical cancer cells suppresses cell proliferation and invasion. Acta Biochim Biophys Sin (Shanghai) 42: 224-229, 2010.

16. Dong Y, Liang G, Yuan B, Yang C, Gao R and Zhou X: MALAT1 promotes the proliferation and metastasis of osteosarcoma cells by activating the PI3K/Akt pathway. Tumour Biol 36: 1477-1486, 2015.

17. Xu S, Sui S, Zhang J, Bai N, Shi Q, Zhang G, Gao S, You Z, Zhan C, Liu F and Pang D: Downregulation of long noncoding RNA MALAT1 induces epithelial-to-mesenchymal transition via the PI3K-AKT pathway in breast cancer. Int J Clin Exp Pathol 8: 4881-4891, 2015.

18. Yang MH, Hu ZY, Xu C, Xie LY, Wang XY, Chen SY and Li ZG: MALAT1 promotes colorectal cancer cell proliferation/migration/invasion via PRKA kinase anchor protein 9. Biochim Biophys Acta 1852: 166-174, 2015.

19. Prat J: Staging classification for cancer of the ovary, fallopian tube, and peritoneum: Abridged republication of guidelines from the international federation of gynecology and obstetrics (FIGO). Obstet Gynecol 126: 171-174, 2015.

20. Livak KJ and Schmittgen TD: Analysis of relative gene expression data using real-time quantitative PCR and the 2(-Delta Delta C(T)) Method. Methods 25: 402-408, 2001.

21. Chen D, Li Y, Su Z, Yu Z, Yu W, Li Y, Gui Y, Yang S and Lai Y: Identification of miR-125a-5p as a tumor suppressor of renal cell carcinoma, regulating cellular proliferation, migration and apoptosis. Mol Med Rep 11: 1278-1283, 2015.

22. Qiu JJ, Lin YY, Ding JX, Feng WW, Jin HY and Hua KQ: Long non-coding RNA ANRIL predicts poor prognosis and promotes invasion/metastasis in serous ovarian cancer. Int J Oncol 46: 2497-2505, 2015

23. Silva JM, Boczek NJ, Berres MW, Ma X and Smith DI: LSINCT5 is over expressed in breast and ovarian cancer and affects cellular proliferation. RNA Biol 8: 496-505, 2011.

24. Qiu JJ, Lin YY, Ye LC, Ding JX, Feng WW, Jin HY, Zhang Y, Li Q and Hua KQ: Overexpression of long non-coding RNA HOTAIR predicts poor patient prognosis and promotes tumor metastasis in epithelial ovarian cancer. Gynecol Oncol 134: 121-128, 2014.

25. Salameh A, Lee AK, Cardó-Vila M, Nunes DN, Efstathiou E, Staquicini FI, Dobroff AS, Marchiò S, Navone NM, Hosoya $\mathrm{H}$, et al: PRUNE2 is a human prostate cancer suppressor regulated by the intronic long noncoding RNA PCA3. Proc Natl Acad Sci USA 112: 8403-8408, 2015.

26. Ding C, Cheng S, Yang Z, Lv Z, Xiao H, Du C, Peng C, Xie H, Zhou L, Wu J and Zheng S: Long non-coding RNA HOTAIR promotes cell migration and invasion via down-regulation of RNA binding motif protein 38 in hepatocellular carcinoma cells. Int J Mol Sci 15: 4060-4076, 2014

27. Tripathi V, Ellis JD, Shen Z, Song DY, Pan Q, Watt AT, Freier SM, Bennett CF, Sharma A, Bubulya PA, et al: The nuclear-retained noncoding RNA MALAT1 regulates alternative splicing by modulating SR splicing factor phosphorylation. Mol Cell 39: 925-938, 2010.

28. Hu L, Wu Y, Tan D, Meng H, Wang K, Bai Y and Yang K: Up-regulation of long noncoding RNA MALAT1 contributes to proliferation and metastasis in esophageal squamous cell carcinoma. J Exp Clin Cancer Res 34: 7, 2015.

29. Hirata H, Hinoda Y, Shahryari V, Deng G, Nakajima K, Tabatabai ZL, Ishii N and Dahiya R: Long Noncoding RNA MALAT1 Promotes Aggressive Renal Cell Carcinoma through Ezh2 and Interacts with miR-205. Cancer Res 75: 1322-1331, 2015. 